\title{
Targeted and synergistic therapy for hepatocellular carcinoma: monosaccharide modified lipid nanoparticles for the co-delivery of doxorubicin and sorafenib
}

This article was published in the following Dove Press journal:

Drug Design, Development and Therapy

\section{Wendu Duan \\ Yan Liu}

Department of Hepatobiliary Surgery, Affiliated Hospital of Hebei University, Baoding, Hebei Province 071000,

People's Republic of China
Correspondence: Yan Liu Department of Hepatobiliary Surgery, Affiliated Hospital of Hebei University, No 648 Dongfengdong Road, Baoding, Hebei Province 071000, People's Republic of China

Email liuyanhebeiu@sina.com
Purpose: Targeted hepatocellular carcinoma therapy was carried out to improve the efficacy of liver cancer treatment. The purpose of this study was to design an $\mathrm{N}$-acetylgalactosamine (NAcGal) modified and $\mathrm{pH}$ sensitive doxorubicin (DOX) prodrug (NAcGal-DOX) for the construction of lipid nanoparticles (LNPs).

Methods: NAcGal-DOX and sorafenib (SOR) co-loaded LNPs were designed and the synergistic effects were evaluated on human hepatic carcinoma (HepG2) cells in vitro and anti-hepatic carcinoma mice model in vivo.

Results: Cellular uptake efficiency of NAcGal modified LNPs was significantly higher than unmodified LNPs. NAcGal modified LNPs showed the most significant inhibition effect among all the samples tested. The results revealed that the LNPs system achieved significant synergistic effects, best tumor inhibition ability and the lowest systemic toxicity.

Conclusion: These results proved that the NAcGal conjugated and $\mathrm{pH}$ sensitive co-delivery nano-system could be a promising strategy for treatment of hepatocellular carcinoma.

Keywords: hepatocellular carcinoma, asialoglycoprotein receptor, N-acetylgalactosamine, $\mathrm{pH}$ sensitive, prodrug

\section{Introduction}

Hepatocellular carcinoma (HCC) remains a major global health problem. ${ }^{1}$ It is the fifth most common cancer and the second leading cause of cancer-related death worldwide. ${ }^{2}$ Currently, due to the majority of patients diagnosed with HCC that are not eligible for surgery, systemic therapy has often been another treatment option for those patients with advanced disease including common therapeutic regimens based on doxorubicin and sorafenib. ${ }^{3-5}$ However, various clinical studies have demonstrated that conventional cytotoxic chemotherapy had low response rates and lacked overall survival benefit. ${ }^{6}$

In HCC patients, doxorubicin (DOX) has the highest activity among chemotherapy agents including gemcitabine and capecitabine, with a response rate of $20 \%$ and a median survival of 4 months. ${ }^{7,8}$ However, the lower response rate and severe side effects like cardiotoxicity and cytotoxicity to normal tissues have hindered its clinical application. ${ }^{9}$ Therefore, targeted drug delivery strategies and targeted drugs have emerged as the most promising fields, and have become research hotspots globally. Current strategies to deliver DOX include using nanoparticles (NPs) with 
long-circulative polyethylene glycol (PEG) and stimuliresponsive materials. ${ }^{10,11}$

Prodrug-based targeted nanoparticulate drug delivery systems have many advantages as platforms for cancer therapy. ${ }^{12}$ Thus, our strategy to address the limitations of DOX is to synthesize N-acetylgalactosamine modified and $\mathrm{pH}$ sensitive DOX prodrug (NAcGal-DOX). $\mathrm{N}$-acetylgalactosamine (NAcGal) targets hepatic cancer cells where asialoglycoprotein receptors (ASGPR) are highly expressed. The $\mathrm{pH}$ sensitivity allows rapid drug release at the tumor site where $\mathrm{pH}$ is lower than 7.4. ${ }^{13,14}$ Recently, studies have designed monosaccharide (galactosamine, NAcGal) and disaccharide (pullulan) decorated nanocarriers for DOX delivery, and achieved liver target in vitro and in vivo based on animal models. ${ }^{14-18}$ However, there are no studies about NAcGal conjugated and $\mathrm{pH}$ sensitive (hydrazine linked) DOX prodrugs.

Targeted drugs for HCC therapy are other effective choices. Sorafenib (SOR) is the only US Food and Drug Administration approved treatment for advanced $\mathrm{HCC}$, achieving modest response rates. ${ }^{19-21}$ It is an oral multikinase inhibitor that suppresses tumor cell proliferation and angiogenesis. ${ }^{22}$ It inhibits various receptors, namely VEGFR1-3, PDGFR-B, c-KIT and Fms-related tyrosine kinase-3, and exerts cytostatic effects to achieve its anticancer activities. ${ }^{23,24}$ In a randomized phase II trial, outcomes have revealed that the combination of SOR and DOX had synergistic effect; specifically, the combination arm had an overall survival of 13.7 months compared to 6.5 months in the DOX group, and progression free survival (PFS) of 6 months in the combination arm vs 2.7 months in the DOX arm. ${ }^{25}$ Therefore, lipid nanoparticles (LNPs) were designed for co-delivery of SOR and NAcGal-DOX to improve their synergy and reduce the side effects.

In this present study, we report the development of multifunctionalized LNPs for HCC targeted delivery of NAcGal-DOX and SOR. NAcGal-DOX conjugates were synthesized with NAcGal decoration, PEG and acid-labile hydrazine links. The LNPs were fabricated by single-step nanoprecipitation. $\mathrm{pH}$ responsiveness of the LNPs was evaluated in vitro by drug release behavior with different pH mediums. Finally, in vitro cell viability assays were carried out on HCC cells (HepG2 cells); and in vivo antitumor activity of drugs loaded LNPs was evaluated in HepG2 tumor xenograft mouse model.

\section{Materials and methods}

\section{Materials}

SOR was purchased from LC Laboratories (Woburn, MA, USA). NAcGal, DOX, polysorbate 80, 1-ethyl-3- (3-dimethylaminopropyl) carbodiimide (EDC), dimethyl sulfoxide (DMSO), fetal bovine serum (FBS), Dulbecco's Modified Eagle's Medium (DMEM), and 3-[4,5-dimethylthiazol-2yl]-2,5 diphenyltetrazolium (MTT) were purchased from Sigma Aldrich (St Louis, MO, USA). Compritol ${ }^{\circledR} 888$ ATO was obtained from Gattefossé (Saint-Priest, Lyon, France). Injectable soya lecithin was obtained from Lipoid $\mathrm{GmbH}$ (Ludwigshafen, Germany). All other chemicals and reagents were of analytical grade or high performance liquid chromatography (HPLC) grade and used without further purification.

\section{Synthesis and characterization of NAcGal-DOX}

NAcGal (1 mmol) was dissolved in $10 \mathrm{~mL}$ of dimethylformamide (DMF) and $\mathrm{NaH}(1 \mathrm{mmol})$ was added as a solid followed by the addition of tertbutyl bromoacetate $(1 \mathrm{mmol})$ in $2 \mathrm{~mL}$ of DMF to get mixture $1 .{ }^{17}$ After stirring at room temperature for 48 hours, hydrazine $(1 \mathrm{mmol})$ and EDC ( $1 \mathrm{mmol}$ ) was added into mixture 1 and stirred for 4 hours in an ice bath to form mixture 2. DOX (1 mmol) was dissolved in DMF $(10 \mathrm{~mL})$ and added into mixture 2 and stirred for another 24 hours, dialyzed against excess ultrapure water for 48 hours to produce NAcGal-DOX (65\% yield). Hydrogen nuclear magnetic resonance $\left({ }^{1} \mathrm{H} \mathrm{NMR}\right)$ of NAcGal-DOX in dimethyl sulfoxide-d6 $(600 \mathrm{mHz})$ is presented, and each proton peak is identified according to the structural formula: $\delta 1.21\left(1,-\mathrm{CH}_{3}\right) ; 2.05(2,-\mathrm{C}-\mathrm{OH}) ; 2.82\left(3,-\mathrm{CH}_{2}-\right) ; 3.11$ $\left(4,-\mathrm{CH}_{2}-\mathrm{O}\right) ; 3.84\left(5,-\mathrm{O}-\mathrm{CH}_{3}\right) ; 4.19(6,-\mathrm{CH}-) ; 4.93$ (7, $\left.-\mathrm{CH}_{2}-\mathrm{C}=\mathrm{O}\right) ; 5.38$ (8, -O-CH-C-); 5.96 (9, -C-CH-C-); $6.91(10,-\mathrm{NH}-\mathrm{CO}-) ; 8.11(11,-\mathrm{NHAc})$ (Figure 1).

\section{Preparation of LNPs}

NAcGal-DOX and SOR loaded LNPs (NAcGal-DOX/SOR LNPs) were prepared via single-step nanoprecipitation (Figure 2). ${ }^{26}$ Briefly, NAcGal-DOX (100 mg), SOR (100 mg), and injectable soya lecithin (1 g) were dissolved in DMSO $(5 \mathrm{~mL})$ as the oil phase. Polysorbate 80 (200 mg) was dispersed in deionized water $(20 \mathrm{~mL})$ to form the water phase. The oil phase was added to the water phase dropwise under gentle stirring (300 rpm). The NAcGal-DOX/SOR LNPs were self-assembled with continuous stirring for 30 minutes at room temperature. For purification, the solution was dialyzed against excess ultrapure water for 2 days (moelcular weight cut off, 3,500 Da).

DOX and SOR loaded LNPs (DOX/SOR LNPs) were prepared by the same procedure using DOX instead of NAcGal-DOX. NAcGal-DOX loaded LNPs (NAcGal-DOX LNPs) were prepared by the same procedure without SOR. SOR loaded LNPs (SOR LNPs) were prepared by the same 


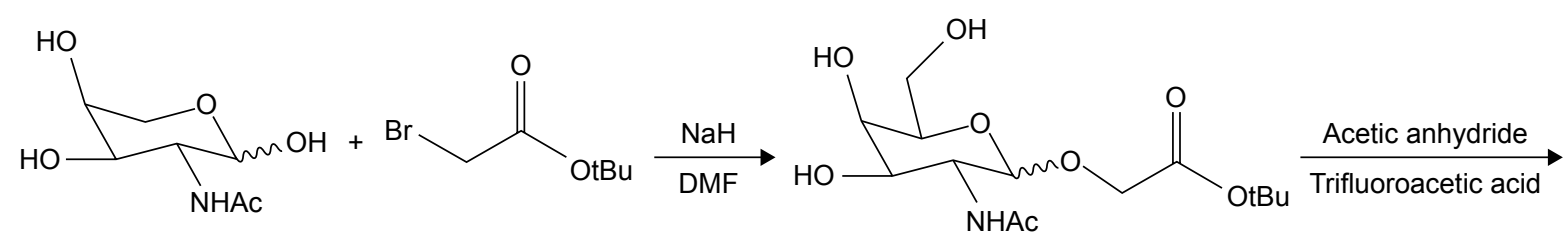

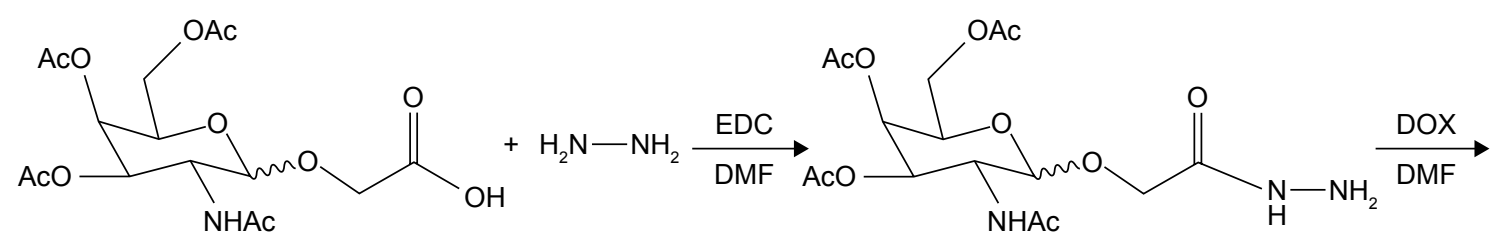

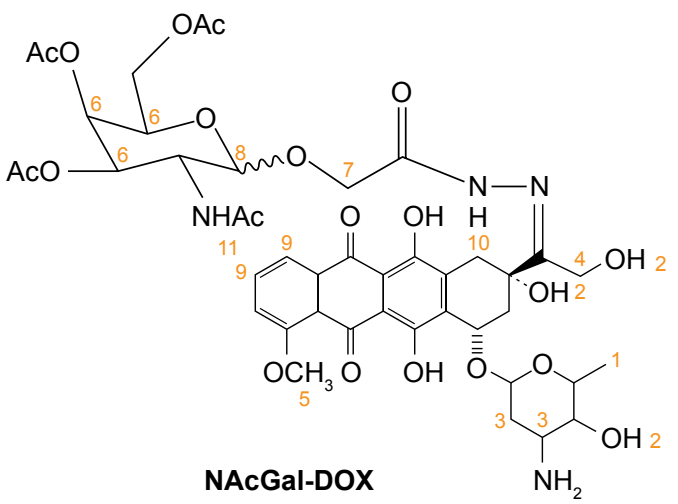

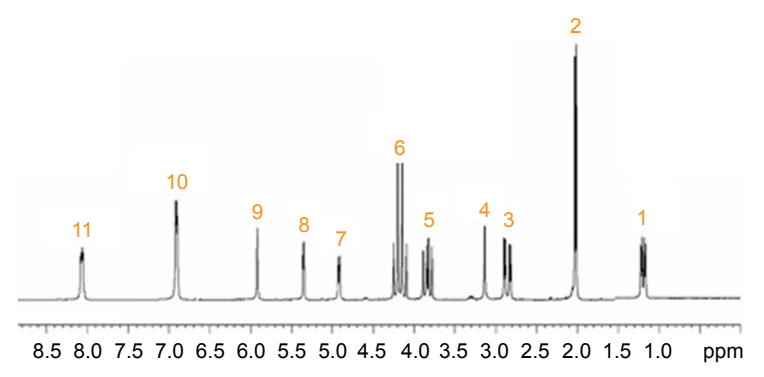

Figure I Synthesis scheme and 'H NMR of NAcGal-DOX in dimethyl sulfoxide-d6.

Abbreviations: DMF, dimethylformamide; DOX, doxorubicin; EDC, I-ethyl-3-(3-dimethylaminopropyl) carbodiimide; 'H NMR, hydrogen nuclear magnetic resonance; NAcGal, N-acetylgalactosamine.

procedure without NAcGal-DOX. Blank LNPs were prepared by the same procedure without NAcGal-DOX and SOR.

\section{Characterization of LNPs}

The particle size and zeta potential were measured by the Nano-ZS apparatus (Malvern Instruments, Malvern, UK). ${ }^{27}$
To evaluate the drug contents and loading efficiency, $5 \mathrm{mg}$ of LNPs were distributed into the mobile phase (acetonitrile/ methanol $/ 1 \%$ acetic acid in a ratio of 35:38:27) and stirred overnight. DOX concentration was determined with UV spectrophotometry (Shimadzu, Kyoto, Japan) at $478 \mathrm{~nm}$ and SOR concentration was evaluated by high-performance liquid

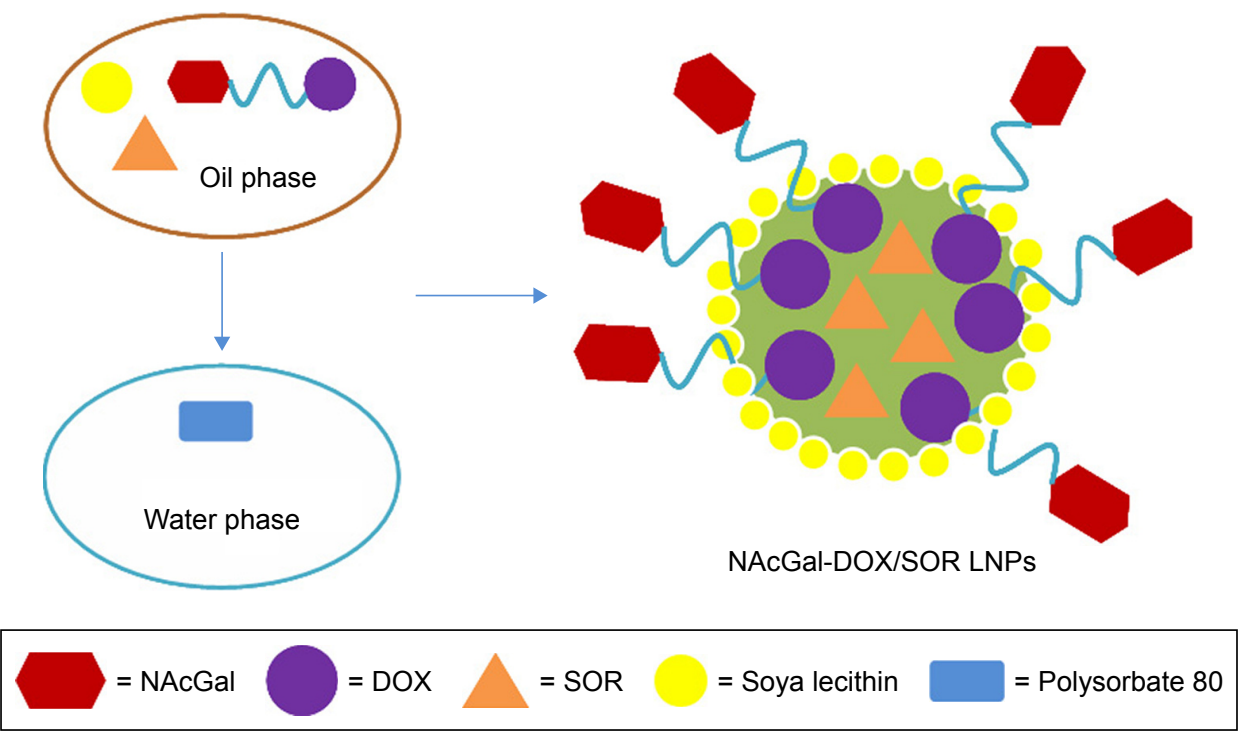

Figure 2 Scheme graph of NAcGal-DOX/SOR LNPs.

Abbreviations: DOX, doxorubicin; LNP, lipid nanoparticle; NAcGal, N-acetylgalactosamine; SOR, sorafenib. 
chromatography (HPLC). The percentage drug content was calculated using the following equations:

$$
\begin{aligned}
& \text { Drug content }(\%)=\frac{\text { Drug weight in the NPs }}{\text { Weight of the NPs }} \times 100 \\
& \text { Loading efficiency }(\%) \\
& =\frac{\text { Residual drug in the NPs }}{\text { Initial feeding amount of drugs }} \times 100
\end{aligned}
$$

\section{In vitro drug release of LNPs}

LNPs was dissolved in $1 \mathrm{~mL}$ of phosphate buffered saline (PBS) in the presence or absence of $10 \%$ mouse serum, and placed in a sealed dialysis tube (molecular weight cut off, $8-10 \mathrm{kDa}) .{ }^{28}$ The dialysis bag was then submerged in $50 \mathrm{~mL}$ tubes (Falcon, BD Labware, NJ, USA) containing $20 \mathrm{~mL}$ sodium phosphate buffer $(50 \mathrm{mM})$ of different $\mathrm{pH}$ (ie, 7.4 and 5.5), and incubated in water-bath at $37^{\circ} \mathrm{C}$ with a shaking rate of $100 \mathrm{rpm}$. Drugs released from the dialysis bags were collected at scheduled time intervals and their amounts were quantified by HPLC as described above.

\section{Cells and animals}

Human liver carcinoma cells (HepG2 and HuH7 cells) were from the American Type Culture Collection (ATCC; Manassas, VA, USA), and the cells were cultured in DMEM with $10 \% \mathrm{FBS}$, at $37^{\circ} \mathrm{C}$ under atmosphere of $5 \% \mathrm{CO}_{2} / 95 \%$ air. BALB/c nude mice (4-6 weeks old, 18-22 g weight) were purchased from Beijing Vital River Experimental Animal Technical Co., Ltd (Beijing, China), and were maintained under specific pathogen-free conditions. All animal experiments complied with the National Institutes of Health guide for the care and use of laboratory animals (NIH Publications No 8023, revised 1978) and approved by the Medical Ethics Committee of Hebei University (HBU-No 1020170607002).

\section{Cellular uptake of LNPs}

Intracellular accumulation assay was used on HepG2 cells to quantitatively determine the cellular uptake of the LNPs. ${ }^{29}$ Coumarin 6 (C6) was applied as a model fluorescent molecule and was loaded into the LNPs by adding C6 to the oil phase during the preparation of the LNPs. After cells were equilibrated with Hank's buffered salt solution (HBSS) at $37^{\circ} \mathrm{C}$ for 1 hour, C6 loaded LNPs were added at concentrations of $200 \mathrm{mg} / \mathrm{mL}$, respectively. The medium was removed after incubation for the determined time, the fluorescence intensity was measured by inversion fluorescence microscope and the picture was captured. Then the cells were washed three times with cold PBS solution and detached with trypsin/EDTA. The cells were centrifuged at $1,500 \mathrm{rpm}, 4^{\circ} \mathrm{C}$ for 5 minutes, the supernatant was discarded, $300 \mu \mathrm{L}$ of PBS was added to re-suspend the cells and injected to a FACSCalibur flow cytometer (Becton, Dickinson and Company, Franklin Lakes, NJ, USA).

\section{In vitro cytotoxicity of LNPs}

The in vitro cytotoxicity of LNPs towards HepG2 and HuH7 cells was evaluated by MTT assay. ${ }^{30}$ Cells were seeded in 96-well plates at a density of 6,000 cells per well in $0.1 \mathrm{~mL}$ DMEM solution and incubated in $5 \% \mathrm{CO}_{2}$ atmosphere at $37^{\circ} \mathrm{C}$ for 24 hours, followed by removing culture medium and then adding $0.1 \mathrm{~mL}$ of NAcGal-DOX/SOR LNPs, DOX/ SOR LNPs, NAcGal-DOX LNPs, SOR LNPs, free DOX and SOR mixture (free DOX/SOR), free DOX, free SOR, and blank LNPs. Cells incubated with $0.1 \mathrm{~mL}$ of PBS were used as control. After 24 hours' incubation, the medium was discarded and $20 \mu \mathrm{L}$ of MTT solution was added to the cells for another 4 hours. The absorbency of the medium solution was measured on a microplate reader at $570 \mathrm{~nm}$. The cell viability was expressed using the following equation:

$$
\text { Cell viability }(\%)=\frac{\text { The absorbency of sample }}{\text { The absorbency of control }} \times 100 \text {. }
$$

\section{In vitro synergistic effects of LNPs}

Half-maximal inhibitory concentration $\left(\mathrm{IC}_{50}\right)$ was then calculated for each sample according to the in vitro cytotoxicity results. Synergistic effects of the systems containing dual drugs can be evaluated by a combination index (CI) analysis based on the Chou and Talalay's method. ${ }^{31,32}$ CI values for DOX and SOR were calculated using the following equation:

$$
\mathrm{CI}=(\mathrm{D})_{\mathrm{DOX}} /\left(\mathrm{D}_{\mathrm{X}}\right)_{\mathrm{DOX}}+(\mathrm{D})_{\mathrm{SOR}} /\left(\mathrm{D}_{\mathrm{X}}\right)_{\mathrm{SOR}}
$$

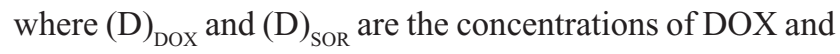
SOR in the combination system at the $\mathrm{IC}_{\mathrm{X}}$ value; $\left(\mathrm{D}_{\mathrm{X}}\right)_{\mathrm{DOX}}$ and $\left(\mathrm{D}_{\mathrm{X}}\right)_{\mathrm{SOR}}$ are $\mathrm{IC}_{\mathrm{X}}$ value of DOX alone and SOR alone.

$\mathrm{CI}_{\mathrm{X}}<1$ represents synergism and $\mathrm{CI}_{\mathrm{X}}>1$ represents antagonism. In this study, $\mathrm{CI}_{50}$ values were applied and the $\mathrm{IC}_{50}$ values were used for calculation.

\section{In vivo tissue distribution of LNPs}

The hepatocellular carcinoma bearing mice model was induced by subcutaneous injection of HepG2 cells ( $10^{7}$ cells suspended in $100 \mu \mathrm{L}$ normal saline) into the right and left 
flanks on the dorsal side of the BALB/c nude mice. ${ }^{33}$ The mice were divided into three groups $(n=6)$ and NAcGalDOX/SOR LNPs, DOX/SOR LNPs, and free DOX/SOR were administered through the tail vein, separately. At predetermined time intervals, mice were sacrificed and the heart, liver, spleen, lung, kidney, stomach, colon, and tumor of mice were collected. The tissues were cut into small pieces and homogenized with physiological saline. After appropriate dilution of supernatants, the content of DOX and SOR was quantified by HPLC as described above.

\section{In vivo therapeutic efficacy of LNPs}

The hepatocellular carcinoma bearing mice were randomly divided into nine groups $(n=6)$ and administered with NAcGal-DOX/SOR LNPs, DOX/SOR LNPs, NAcGal-DOX LNPs, SOR LNPs, free DOX/SOR, free DOX, free SOR, blank LNPs, and 0.9\% normal saline, respectively, through the tail vein on Days 0, 3, and 6. ${ }^{34}$ Tumor volumes were calculated according to the following equation:

$$
\text { Tumor volume }\left(\mathrm{mm}^{3}\right)=\frac{\mathrm{L} \times \mathrm{W}^{2}}{2}
$$

where $\mathrm{L}$ is the longest and $\mathrm{W}$ is the shortest tumor diameter (mm).

Then mice were sacrificed and tumors were harvested at the completion of the experiment. Tumor weights were measured, and tumor growth inhibition ratio was calculated using the following equation:

Tumor inhibition ratio ( $\%)$

(Tumor weight of saline treated group$=\frac{\text { Tumor weight of drug treated group) }}{\text { Tumor weight of saline treated group }} \times 100$.

\section{Blood collection and analysis}

The hepatocellular carcinoma bearing mice were divided into three groups $(n=6)$ and NAcGal-DOX/SOR LNPs,
DOX/SOR LNPs, free DOX/SOR, and 0.9\% normal saline were administered through the tail vein, separately. ${ }^{35}$ On Day 7 after injection, the blood was collected into heparinized tubes through cardiac puncture under anesthesia and animals were euthanized via $\mathrm{CO}_{2}$ overdose. Blood was centrifuged at $15,000 \mathrm{rpm}$ for 20 minutes at $4^{\circ} \mathrm{C}$ to isolate plasma, which was immediately flash frozen on liquid nitrogen until processing. Plasma was assayed for blood enzymes as biomarkers for different tissue toxicity using assay kits, namely, lactate dehydrogenase (LDH), alanine transaminase (ALT), and creatine phosphokinase (CPK).

\section{Evaluation of toxicity during the repeated treatment}

The hepatocellular carcinoma bearing mice were divided into three groups $(n=6)$ and NAcGal-DOX/SOR LNPs, DOX/ SOR LNPs, free DOX/SOR, and 0.9\% normal saline were administered through the tail vein, separately. The physical conditions and body weight change of mice were monitored for 3 weeks. ${ }^{30}$

\section{Results}

\section{Characterization of LNPs}

The particle size, zeta potential, drug content and loading efficiency of LNPs were measured (Table 1). The size of NAcGal-DOX/SOR LNPs is $121 \mathrm{~nm}$, which is larger than that of DOX/SOR LNPs (102 nm). Sizes of NAcGal-DOX/ SOR LNPs and NAcGal-DOX LNPs are the same. DOX/ SOR LNPs, SOR LNPs and blank LNPs have similar sizes. The polymer dispersity index (PDI) of all LNPs was below 0.2 . Drug contents of drugs loaded LNPs are over $80 \%$.

\section{In vitro drug release}

DOX releases from NAcGal-DOX contained LNPs at $\mathrm{pH} 5.5$ are faster than $\mathrm{pH} 7.4$ (Figure 3A). For NAcGal-DOX/SOR, over $80 \%$ release of DOX was achieved at 36 hours in acidic condition ( $\mathrm{pH}$ 5.5). In contrast, in a neutral environment the release reached $80 \%$ at 48 hours. The SOR releases were not affected by the $\mathrm{pH}$ conditions (Figure 3B). The release

Table I Characterization of LNPs (mean \pm SD, $n=3$ )

\begin{tabular}{|c|c|c|c|c|c|c|c|}
\hline \multirow[t]{2}{*}{ LNPs } & \multirow{2}{*}{$\begin{array}{l}\text { Particle } \\
\text { size }(\mathbf{n m})\end{array}$} & \multirow{2}{*}{$\begin{array}{l}\text { Size distribution } \\
\text { (PDI) }\end{array}$} & \multirow{2}{*}{$\begin{array}{l}\text { Zeta potential } \\
(\mathrm{mV})\end{array}$} & \multicolumn{2}{|c|}{ Drug content (\%) } & \multicolumn{2}{|c|}{ Loading efficiency (\%) } \\
\hline & & & & DOX & SOR & DOX & SOR \\
\hline NAcGal-DOX/SOR LNPs & $121.2 \pm 3.5$ & $0.16 \pm 0.03$ & $-37.4 \pm 3.6$ & $80.7 \pm 2.9$ & $83.2 \pm 3.3$ & $5.6 \pm 0.5$ & 4. $1 \pm 0.4$ \\
\hline DOX/SOR LNPs & $102.3 \pm 2.9$ & $0.13 \pm 0.02$ & $-24.1 \pm 2.9$ & $82.2 \pm 3.1$ & $82.7 \pm 3.1$ & $7.8 \pm 0.8$ & $6.3 \pm 0.6$ \\
\hline NAcGal-DOX LNPs & $120.8 \pm 3.2$ & $0.15 \pm 0.02$ & $-35.9 \pm 3.2$ & $81.6 \pm 2.7$ & $\mathrm{~N} / \mathrm{A}$ & $6.1 \pm 0.4$ & N/A \\
\hline SOR LNPs & $100.9 \pm 2.7$ & $0.13 \pm 0.02$ & $-23.6 \pm 3.1$ & $\mathrm{~N} / \mathrm{A}$ & $84.3 \pm 3.5$ & $\mathrm{~N} / \mathrm{A}$ & $7.2 \pm 0.5$ \\
\hline Blank LNPs & $101.3 \pm 2.5$ & $0.12 \pm 0.01$ & $-22.1 \pm 2.5$ & $N / A$ & N/A & $\mathrm{N} / \mathrm{A}$ & $\mathrm{N} / \mathrm{A}$ \\
\hline
\end{tabular}

Abbreviations: DOX, doxorubicin; LNP, lipid nanoparticle; NAcGal, N-acetylgalactosamine; PDI, polymer dispersity index; SOR, sorafenib. 

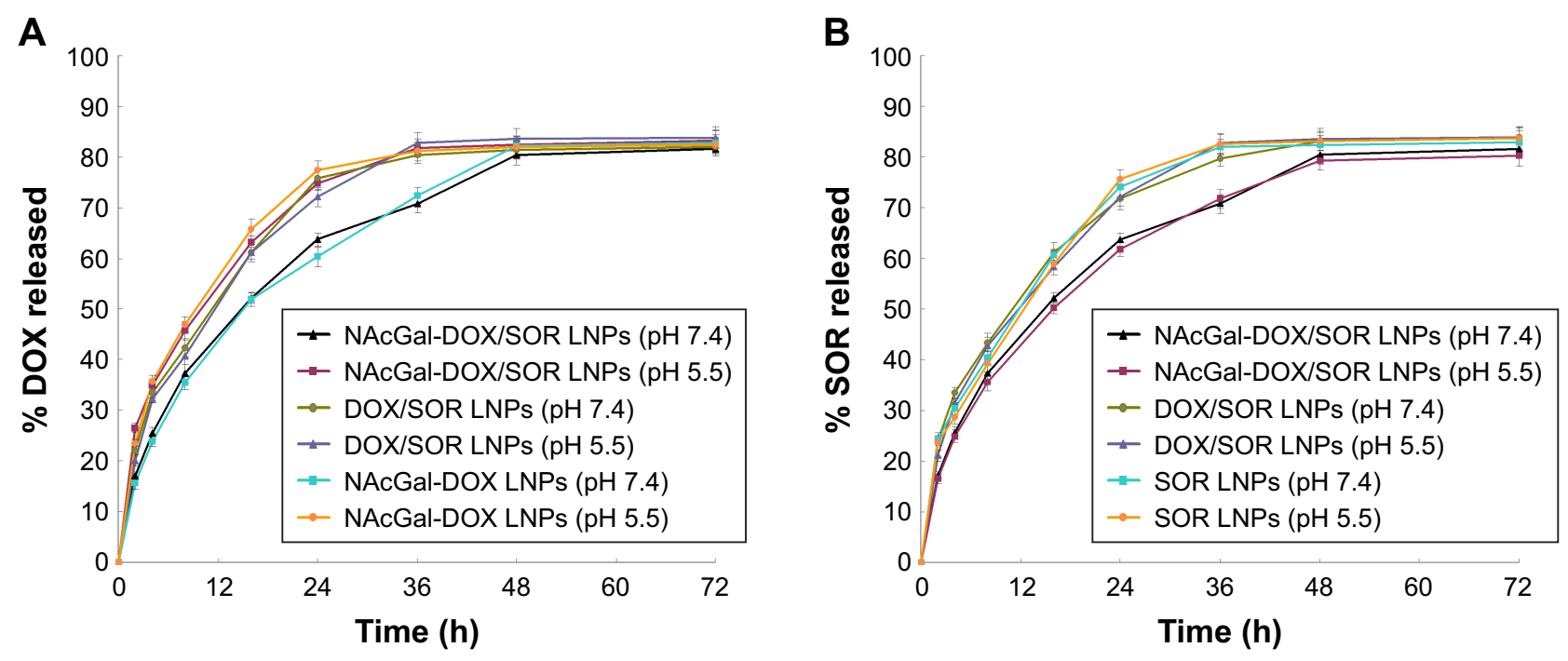

Figure 3 In vitro DOX (A) and SOR (B) releases from NAcGal-DOX contained LNPs at pH 5.5 and pH 7.4.

Note: Data represent mean \pm SD $(n=3)$.

Abbreviations: DOX, doxorubicin; LNP, lipid nanoparticle; NAcGal, N-acetylgalactosamine; SOR, sorafenib.

curves of the same system in $\mathrm{pH} 7.4$ and 5.5 followed the same behavior. The SOR release from NAcGal-DOX/SOR LNPs was slower than that from DOX/SOR LNPs and SOR LNPs.

\section{Cellular uptake of LNPs}

Cellular uptake of the LNPs increased with time (Figure 4). Cellular uptake efficiency of NAcGal modified LNPs was significantly higher than unmodified LNPs $(P<0.05)$. After 9 hours of incubation, over $70 \%$ of cell uptake was achieved by NAcGal-DOX/SOR LNPs.

\section{In vitro cytotoxicity of LNPs}

NAcGal modified LNPs showed the most significant inhibition effect among all the samples tested $(P<0.05)$. Dual drug loaded LNPs exhibited higher efficiency than single drug loaded LNPs $(P<0.05)$. The LNP samples had better performances than their free drug counterparts $(P<0.05)$. In addition, data revealed blank NPs that do not contain drugs showed negligible toxicity (Figure 5). The in vitro cytotoxicity results indicated that DOX and SOR released from LNPs could play an enhanced anti-tumor effect.

\section{In vitro synergistic effects of LNPs}

To select the suitable DOX and SOR amount loaded in the LNPs to get the best synergism effect, $\mathrm{CI}_{50}$ values were calculated according to the $\mathrm{IC}_{50}$ values of NAcGal-DOX/SOR LNPs, NAcGal-DOX LNPs, and SOR LNPs (Table 2).
When DOX:SOR ratios were between 10:1 and 1:1, NAcGalDOX/SOR LNPs showed synergistic effects. The best synergistic effect was achieved at the weight ratio of 2:1 (DOX:SOR). So the DOX and SOR loaded in LNPs is determined as 2:1.

\section{In vivo tissue distribution of LNPs}

In vivo tissue distribution of NAcGal-DOX/SOR LNPs, DOX/SOR LNPs, and free DOX/SOR was investigated in hepatocellular carcinoma bearing mice model (Figure 6). After 48 hours of administration, DOX and SOR distributions of NAcGal-DOX/SOR LNPs were higher in the tumor tissue than DOX/SOR LNPs $(P<0.05)$. DOX/SOR LNPs showed better tumor tissue distribution than free DOX/ SOR $(P<0.05)$. At 2 hours post injection, free DOX/SOR showed higher accumulation in heart and kidney than LNP formulas $(P<0.05)$.

\section{In vivo therapeutic efficacy of LNPs}

In vivo antitumor efficiency of LNPs evaluated in hepatocellular carcinoma bearing BALB/c mice (Figure 7) was stronger than free drug(s), which showed modest anti-cancer activity with tumor growth inhibition ratios of $17 \%-44 \%$ (Table 3). Saline-treated mice displayed a rapid increase in tumor size during the experiment. Among all the groups, NAcGal-DOX/SOR LNPs exhibited the most potent antitumor activity. The tumor growth was inhibited with a tumor growth inhibition ratio as high as $87 \%$. 
A
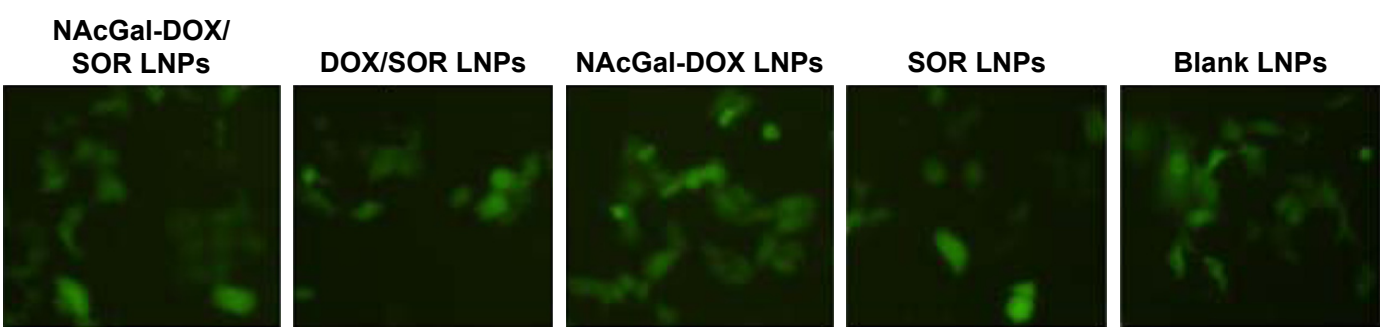

B

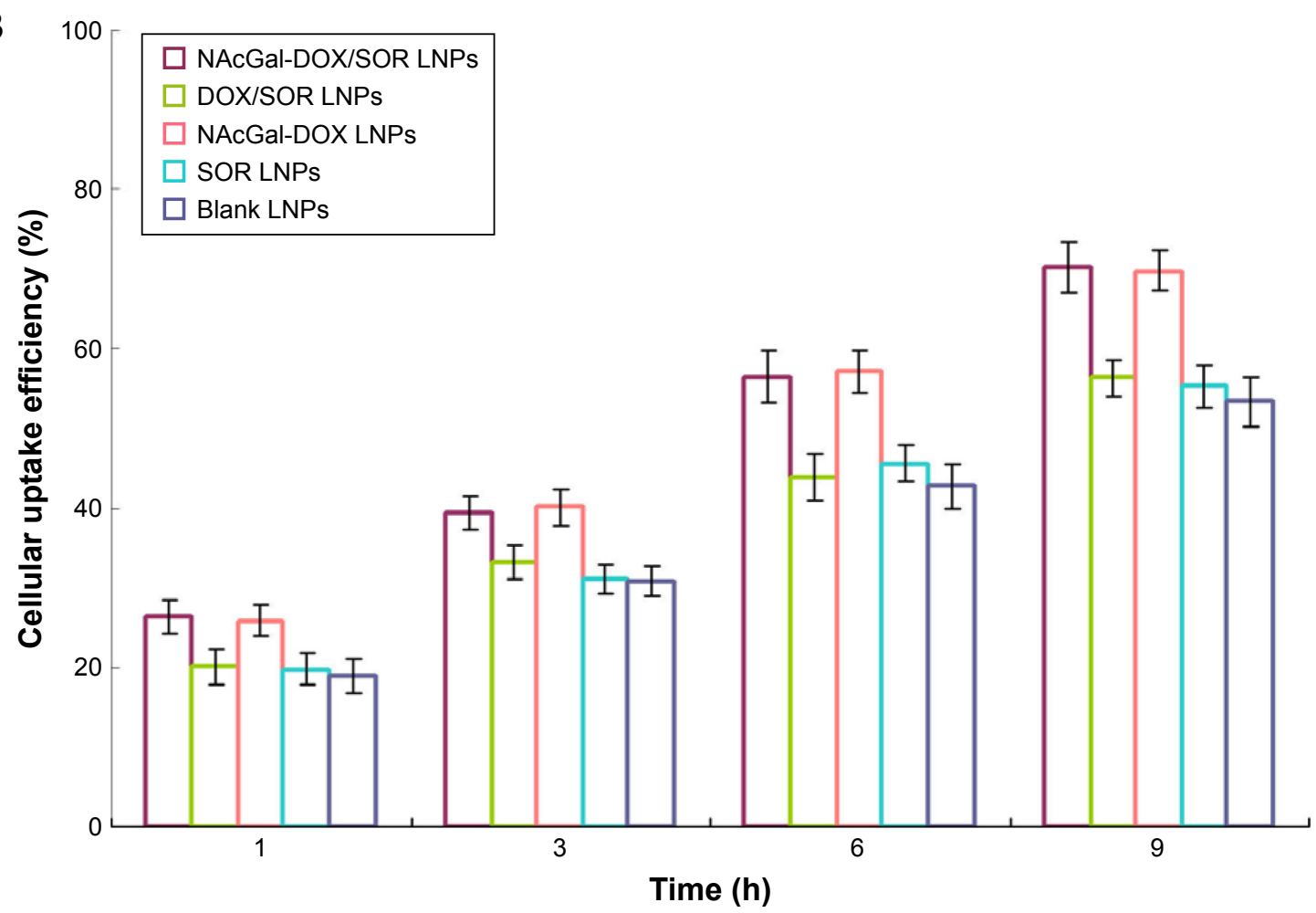

Figure 4 Cellular uptake efficiency of the LNPs evaluated on HepG2 cells, fluorescence images (A) and flow cytometry (B).

Note: Data represent mean $\pm S D(n=3)$.

Abbreviations: DOX, doxorubicin; LNP, lipid nanoparticle; NAcGal, N-acetylgalactosamine; SOR, sorafenib.
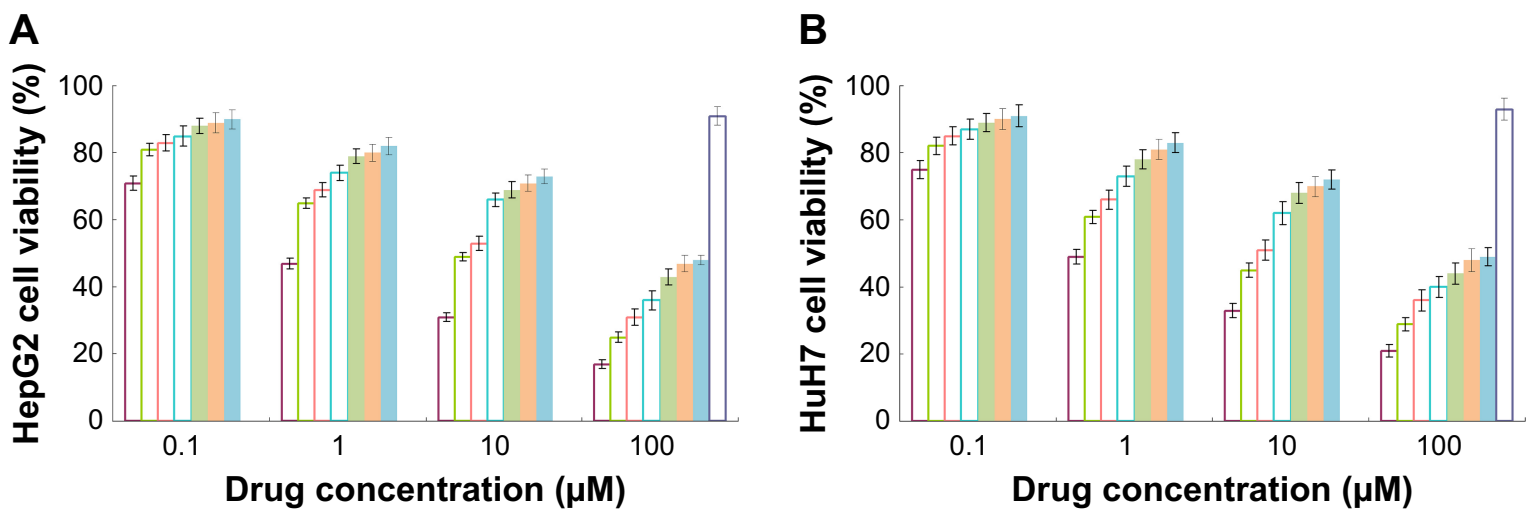

\begin{tabular}{|c|c|c|c|}
\hline Free & $\begin{array}{l}\square \text { DOX/SC } \\
\text { Free DO }\end{array}$ & $\begin{array}{l}\square \text { NAcGal-DOX LNPs } \\
\text { Free SOR }\end{array}$ & \\
\hline
\end{tabular}

Figure 5 In vitro cytotoxicity of LNPs evaluated on HepG2 (A) and HuH7 (B) cells by MTT assay.

Note: Data represent mean \pm SD $(n=3)$.

Abbreviations: DOX, doxorubicin; LNP, lipid nanoparticle; NAcGal, N-acetylgalactosamine; SOR, sorafenib. 
Table $2 \mathrm{IC}_{50}$ and $\mathrm{Cl}_{50}$ values of LNPs with different DOX and SOR content (mean $\pm S D, n=3$ )

\begin{tabular}{|c|c|c|c|c|}
\hline LNPs & $\begin{array}{l}\text { DOX:SOR } \\
\text { (w:w) }\end{array}$ & $\begin{array}{l}\text { IC }{ }_{50} \text { DOX } \\
(\mu \mathrm{M})\end{array}$ & $\begin{array}{l}\text { IC } \text { Fo }_{50} \text { SOR } \\
(\mu \mathrm{M})\end{array}$ & $\mathrm{CI}_{50}$ \\
\hline NAcGal-DOX LNPs & N/A & $19.52 \pm 1.65$ & N/A & $\mathrm{N} / \mathrm{A}$ \\
\hline SOR LNPs & $\mathrm{N} / \mathrm{A}$ & $\mathrm{N} / \mathrm{A}$ & $51.36 \pm 2.75$ & $\mathrm{~N} / \mathrm{A}$ \\
\hline NAcGal-DOX/SOR LNPs & $10: 1$ & $15.23 \pm 1.23$ & $1.52 \pm 1.65$ & 0.81 \\
\hline NAcGal-DOX/SOR LNPs & $5: 1$ & $12.68 \pm 1.04$ & $2.54 \pm 1.65$ & 0.70 \\
\hline NAcGal-DOX/SOR LNPs & $2: 1$ & $1.96 \pm 0.08$ & $0.98 \pm 0.06$ & 0.11 \\
\hline NAcGal-DOX/SOR LNPs & $\mathrm{I}: \mathrm{I}$ & $11.36 \pm 0.89$ & $11.36 \pm 0.69$ & 0.80 \\
\hline NAcGal-DOX/SOR LNPs & $\mathrm{I}: 2$ & $12.21 \pm 1.52$ & $24.42 \pm 2.21$ & 1.10 \\
\hline NAcGal-DOX/SOR LNPs & 1:5 & $9.17 \pm 0.46$ & $45.85 \pm 3.17$ & 1.36 \\
\hline NAcGal-DOX/SOR LNPs & $\mathrm{I}: 10$ & $5.92 \pm 0.31$ & $59.23 \pm 3.61$ & 1.46 \\
\hline
\end{tabular}

Abbreviations: DOX, doxorubicin; $I_{50}$, inhibitory concentration; LNP, lipid nanoparticle; NAcGal, N-acetylgalactosamine; SOR, sorafenib; w:w, weight:weight.

\section{Blood analysis}

Blood enzyme levels were measured serially once a week over the 3-week course of treatment (Figure 8). Although the differences in blood enzyme activities between the treatment groups were not statistically significant $(P>0.05)$, the trends in blood enzyme levels consistently suggested lower toxicity of LNP treatment relative to free DOX/SOR therapy in tumor models. Free DOX/SOR treatment resulted in an increase in LDH, ALT, and CPK.

\section{Toxicity during the repeated treatment \\ Toxicity of LNPs was studied in hepatocellular carcinoma bearing mice. As shown in Figure 9, the mice treated with free drugs led to over $20 \%$ body weight loss on Day 21. In contrast, the LNP administration group showed no obvious changes in body weight. This suggests that all of the LNP formulations were well tolerated. In contrast, decrease of body weights in free drugs treated mice could be the evidence of the toxicity of the systems.}

\section{Discussion}

In the present study, a novel NAcGal conjugated and $\mathrm{pH}$ sensitive LNP system is applied for treatment of hepatocellular carcinoma. At the beginning of this study, NAcGal modified and $\mathrm{pH}$ sensitive PEGylated DOX prodrug conjugate was synthesized. In order to achieve the synergistic effects of DOX and SOR on hepatocellular carcinoma, LNPs were successfully prepared by single-step nanoprecipitation. ${ }^{36}$ The size of
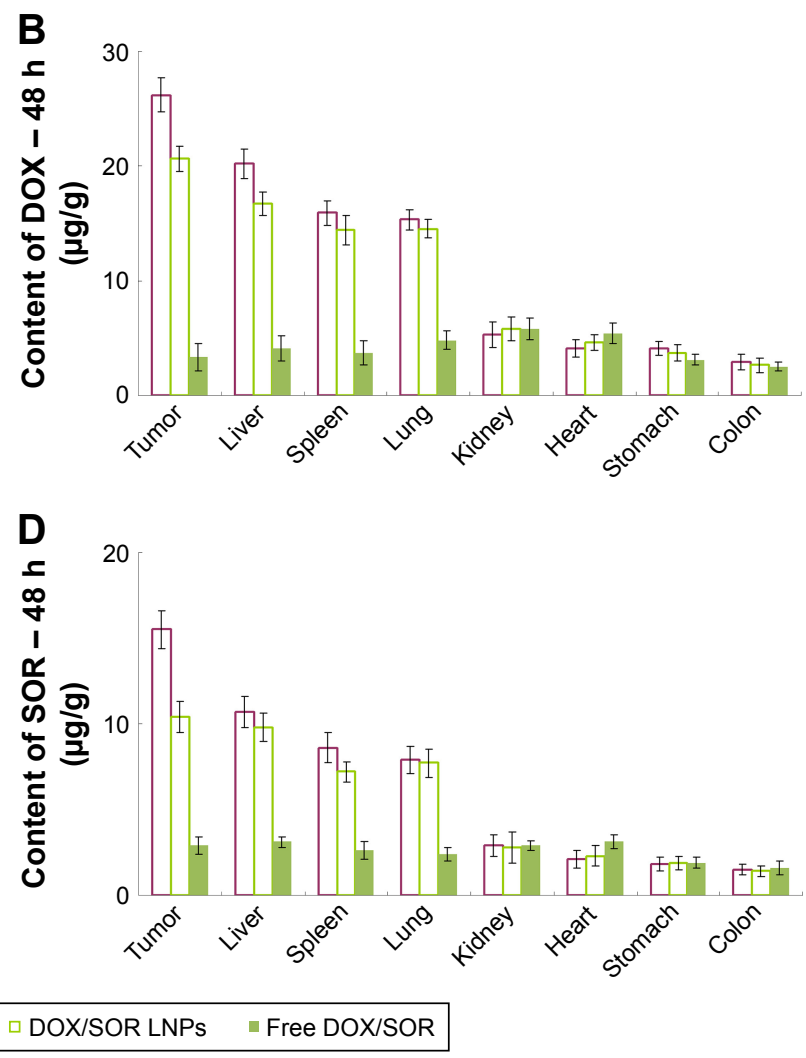

Figure 6 In vivo DOX tissue distribution of NAcGal-DOX/SOR LNPs, DOX/SOR LNPs, and free DOX/SOR investigated in hepatocellular carcinoma bearing mice at 2 hours $(\mathbf{A})$ and 48 hours (B) post administration. In vivo SOR tissue distribution of NAcGal-DOX/SOR LNPs, DOX/SOR LNPs, and free DOX/SOR investigated in hepatocellular carcinoma bearing mice at 2 hours (C) and 48 hours (D) post administration.

Note: Data represent mean \pm SD $(n=6)$.

Abbreviations: DOX, doxorubicin; LNP, lipid nanoparticle; NAcGal, N-acetylgalactosamine; SOR, sorafenib. 


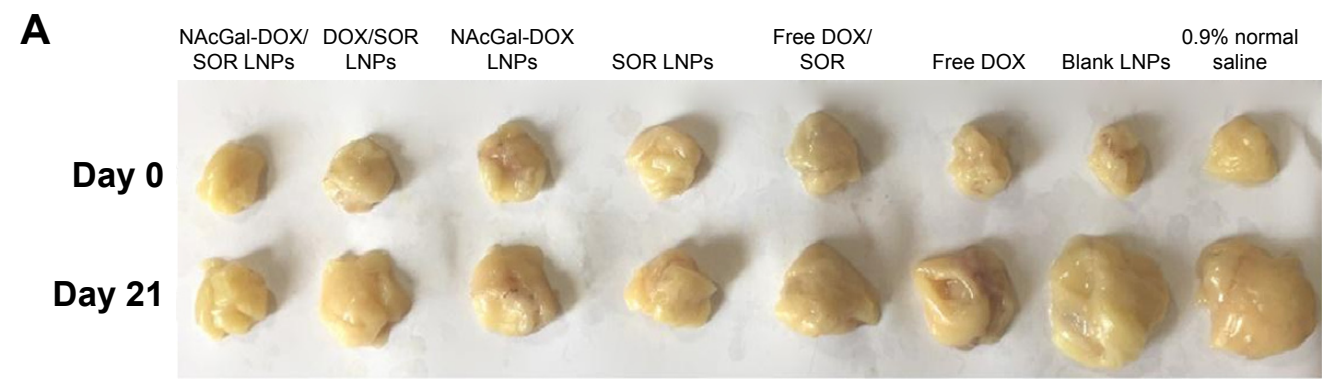

B

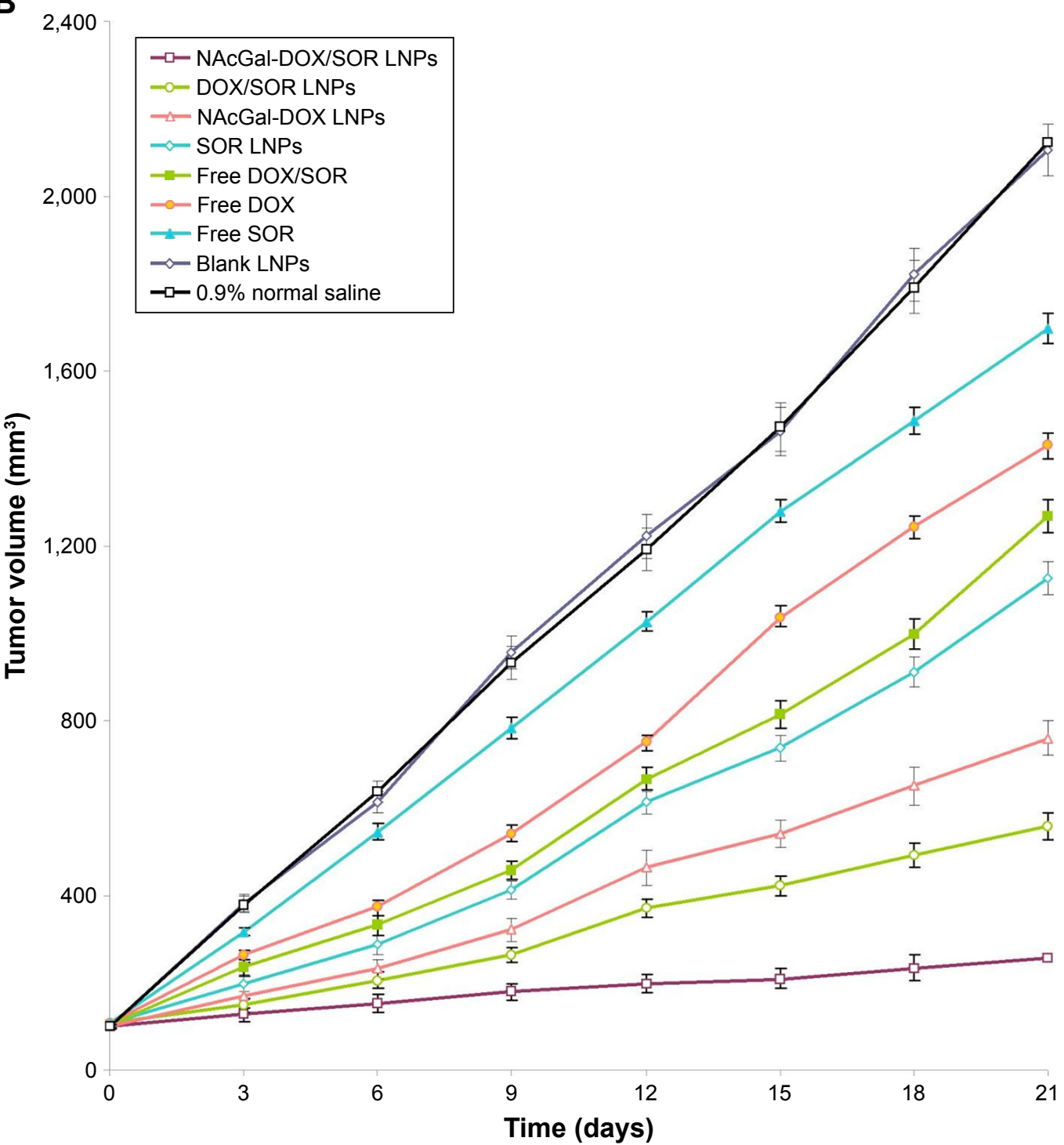

Figure $7 \mathrm{In}$ vivo therapeutic efficacy of LNPs in terms of changes of tumor volume (A) and tumor burden (B) evaluated in hepatocellular carcinoma bearing BALB/c mice. Note: Data represent mean $\pm S D(n=6)$.

Abbreviations: DOX, doxorubicin; LNP, lipid nanoparticle; NAcGal, N-acetylgalactosamine; SOR, sorafenib.

Table 3 Tumor inhibition ratio of LNPs and free drugs (mean \pm SD, $n=6$ )

\begin{tabular}{llllllll}
\hline Systems & $\begin{array}{l}\text { NAcGal-DOX/ } \\
\text { SOR LNPs }\end{array}$ & $\begin{array}{l}\text { DOX/SOR } \\
\text { LNPs }\end{array}$ & $\begin{array}{l}\text { NAcGal-DOX } \\
\text { LNPs }\end{array}$ & SOR LNPs & Free DOX/SOR & Free DOX & Free SOR \\
\hline Tumor inhibition ratio (\%) & $86.9 \pm 3.8$ & $72.5 \pm 3.9$ & $63.7 \pm 3.2$ & $49.1 \pm 2.7$ & $44.3 \pm 2.3$ & $30.6 \pm 1.8$ & $17.1 \pm 0.9$ \\
\hline
\end{tabular}

Abbreviations: DOX, doxorubicin; LNP, lipid nanoparticle; NAcGal, N-acetylgalactosamine; SOR, sorafenib. 

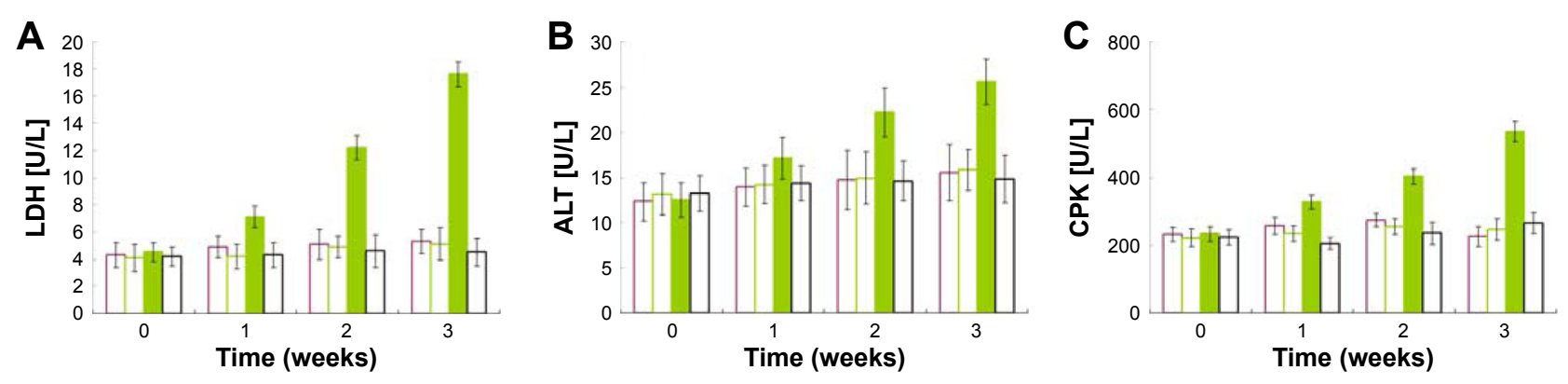

$\square$ NAcGal-DOX/SOR LNPs $\square$ DOX/SOR LNPs $\quad$ Free DOX/SOR $\square 0.9 \%$ normal saline

Figure 8 Blood enzyme levels measured once a week over 3-week course of treatment.

Notes: Plasma was assayed for blood enzymes using assay kits, namely, LDH (A), ALT (B), and CPK (C). Data represent mean \pm SD ( $n=6$ )

Abbreviations: ALT, alanine transaminase; CPK, creatine phosphokinase; DOX, doxorubicin; LDH, lactate dehydrogenase; LNP, lipid nanoparticle; NAcGal, $\mathrm{N}$-acetylgalactosamine; SOR, sorafenib.

blank LNPs and SOR LNPs was about $100 \mathrm{~nm}$, with narrow polydispersity indexes lower than 0.2 . This could be evidence that the loading of the drug has no obvious effect on the size of the LNPs. Particle size has a great impact on the in vitro and in vivo efficiency of the NPs, including prolonged blood circulation time and mediated targeted effect. Drug contents of all LNPs have no significant difference. These results indicated that LNPs had good drug entrapment capacity.

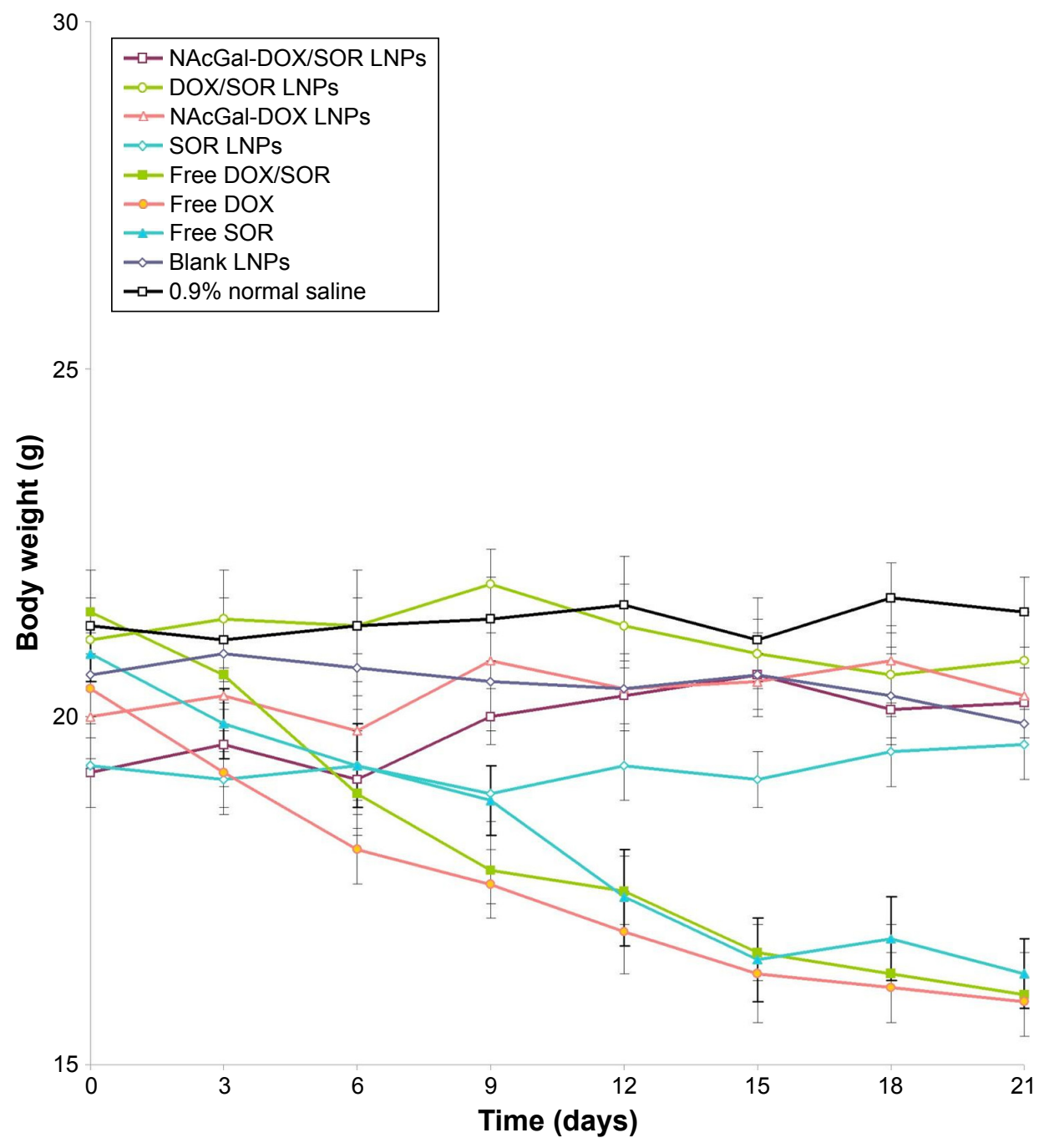

Figure 9 Toxicity of LNPs in terms of body weight loss studied in hepatocellular carcinoma bearing mice.

Note: Data represent mean $\pm S D(n=6)$.

Abbreviations: DOX, doxorubicin; LNP, lipid nanoparticle; NAcGal, N-acetylgalactosamine; SOR, sorafenib. 
In vitro drug release study of LNPs was investigated at $\mathrm{pH} 7.4$ and 5.5. ${ }^{37} \mathrm{In}$ acidic media, the release of DOX was faster than that in the neutral environment. This may be because the $\mathrm{pH}$ sensitive hydrazone bonds could cleave much more easily in the lower $\mathrm{pH}$ and the degradation of the LNPs occurs predominantly via hydrazone bond cleavage and released the DOX loaded in the LNPs. The $\mathrm{pH}$ value of the bloodstream is $\sim 7.4$, while the existing tumoral $\mathrm{pH}$ and that of endocytic compartments of the cells generally range from 4 to $6 .{ }^{38}$ This difference in $\mathrm{pH}$ value makes $\mathrm{pH}$-triggered drug release possible. The DOX release rate from LNPs increased when $\mathrm{pH}$ was lowered. These findings suggest that the weak acidic condition of tumor tissue is favorable for release of DOX.$^{28}$ Further, upon internalization into tumor cells via endocytosis, the low $\mathrm{pH}$ of the phagolysosomal system will facilitate more rapid release of DOX due to lower $\mathrm{pH}$. The SOR release from NAcGal-DOX/SOR LNPs was slower than that from DOX/SOR LNPs and SOR LNPs. In vitro drug release of the LNPs may be controlled by erosion, corrosion, and diffusion processes ${ }^{39}$ Drug depot effects could be achieved by the carriers, which could lead to the sustained release of hydrophobic drugs. The more sustained SOR release behavior from NAcGal-DOX/SOR LNPs than from DOX/SOR LNPs might be explained by the modification of NAcGal ligands that decelerated the release of drugs. Once within the endosomal compartments, the drugs can be released from the LNPs and induce their toxic impacts. In addition, in the presence of serum, the release of drugs was not affected, indicating the stability of LNPs in circulation.

The in vitro cellular uptake research could provide some circumstantial evidence to display the advantages of the NP formulation entering the cancer cells. Cellular uptake efficiency of NAcGal modified LNPs was significantly higher than other LNPs. This could be attributed to enhanced cancer cell specific adherence of the NAcGal ligands to the liver cancer cell membrane. The improved activity and penetration of drugs delivered with NAcGal modified LNPs can be made use of to improve the efficacy of the standard drug dose, attenuate side effects, and overcome drug resistance. ${ }^{29}$

In order to verify the enhanced anticancer effect of the LNPs, the proliferation inhibition of LNPs was tested against HepG2 and HuH7 cells. Free drug and drug mixtures were used as the controls. The cell proliferation inhibition efficacy of all the samples exhibited a strongly dose-dependent pattern. Higher cytotoxicity of the drug-loaded LNPs than free drug indicated that LNP delivery systems can enhance cytotoxicity in vitro. It is encouraging that the anti-tumor activity of NAcGal-DOX/SOR LNPs was obviously higher than the other groups, especially in the range of higher drug concentration. To validate the synergistic effect of drugs coloaded NPs on HepG2 cells, the CI was further determined using the isobologram equation of Chou and Talalay. ${ }^{40}$ NAcGal-DOX/SOR LNPs displayed an overall CI value $<1$ when the DOX:SOR ratio was between 10:1 and 1:1. In the ratio of 2:1, the combined anti-tumor effect of the dual drug-loaded LNPs was the best and could develop the ability of the drugs to the largest extent, suggesting the best DOX:SOR ratio in the LNP formulations.

In vivo drug distribution of LNPs was higher in tumor tissue and lower in the heart and kidney, which could decrease side effects during tumor therapy. Conversely, drug solution samples are mainly distributed in heart and kidney. This may lead to systemic toxicity. Higher distribution of drugs in LNPs in tumors than in other tissues, especially at 48 hours of testing, might be due to the sustained release behavior and targeted ability of the LNPs, thus prolonging blood circulation time and better targeting the tumor site. Higher accumulation of NAcGal-DOX/SOR LNPs than DOX/SOR LNPs was observed in the tumor tissue. This could be evidence that the NAcGal ligands would bring about the target ability to the LNP system. Toxicity of LNPs was studied in hepatocellular carcinoma bearing mice in terms of body weight changes. On Day 21, mice treated with LNPs showed no obvious changes in body weight, while free drugs led to over $20 \%$ body weight loss. The decrease of body weight in free drug treated mice is likely due to the toxicity caused by free DOX and SOR distributed to normal organs. Considering the results along with the blood enzyme level analysis and tissue distribution, these LNPs groups could be indicative of tumor cell death and not necessarily nonspecific tissue damage.

In vivo antitumor efficiency of LNPs was evaluated in $\mathrm{BALB} / \mathrm{c}$ mice bearing hepatocellular carcinoma. The HepG2 cell line is an aggressive cancer cell line with a high proliferation rate, and saline-treated mice displayed a rapid increase in tumor size during the experiment. Free drug(s) showed modest anti-cancer activity, while stronger therapeutic responses were observed in LNP treated mice. NAcGal-DOX/SOR LNPs exhibited the most potent antitumor activity among all the groups; the tumor growth was almost completely inhibited. The results revealed that the higher anti-tumor efficiency of drugs after co-loading in NAcGal-DOX/SOR LNPs than in DOX/SOR LNPs is related to the targeted ability of NAcGal. Also, this high therapeutic efficacy is consistent with the strong synergy between DOX and SOR. The facilitated co-delivery of DOX and SOR by DOX/SOR LNPs also plays an important role as DOX/SOR 
LNPs were much more active than the free drug combination (free DOX/SOR) in antitumor activity.

\section{Conclusion}

In summary, this research provides a solution to formulate a specific NAcGal modified $\mathrm{pH}$ sensitive LNPs system for the co-delivery of DOX and SOR for combination hepatocellular carcinoma chemotherapy. The LNP system achieved significant synergistic effects, best tumor inhibition ability and the lowest systemic toxicity. These results proved that the NAcGal conjugated and $\mathrm{pH}$ sensitive co-delivery nano-system could be a promising strategy for treatment of hepatocellular carcinoma.

\section{Disclosure}

The authors report no conflicts of interest in this work.

\section{References}

1. Nordenstedt H, White DL, El-Serag HB. The changing pattern of epidemiology in hepatocellular carcinoma. Dig Liver Dis. 2010;42(Suppl 3): S206-S214.

2. Jemal A, Bray F, Center MM, Ferlay J, Ward E, Forman D. Global cancer statistics. CA Cancer J Clin. 2011;61(2):69-90.

3. $\mathrm{Sj} \mathrm{Y}$. A concise review of updated guidelines regarding the management of hepatocellular carcinoma around the world: 2010-2016. Clin Mol Hepatol. 2016;22(1):7-17.

4. Yeo W, Mok TS, Zee B, et al. A randomized phase III study of doxorubicin versus cisplatin/interferon alpha-2b/doxorubicin/fluorouracil (PIAF) combination chemotherapy for unresectable hepatocellular carcinoma. J Natl Cancer Inst. 2005;97(20):1532-1538.

5. Lencioni R, Llovet JM, Han G, et al. Sorafenib or placebo plus TACE with doxorubicin-eluting beads for intermediate stage HCC: The SPACE trial. J Hepatol. 2016;64(5):1090-1098.

6. Thomas MB, O’Beirne JP, Furuse J, Chan AT, Abou-Alfa G, Johnson P. Systemic therapy for hepatocellular carcinoma: cytotoxic chemotherapy, targeted therapy and immunotherapy. Ann Surg Oncol. 2008;15(4): 1008-1014.

7. Patt YZ, Hassan MM, Aguayo A, et al. Oral capecitabine for the treatment of hepatocellular carcinoma, cholangiocarcinoma, and gallbladder carcinoma. Cancer. 2004;101(3):578-586.

8. Lombardi G, Zustovich F, Farinati F, et al. Pegylated liposomal doxorubicin and gemcitabine in patients with advanced hepatocellular carcinoma: results of a phase 2 study. Cancer. 2011;117(1): 125-133.

9. Dong L, Xia S, Wu K, et al. A pH/enzyme-responsive tumorspecific delivery system for doxorubicin. Biomaterials. 2010;31(24): 6309-6316.

10. Zhou H, Fan Z, Deng J, et al. Hyaluronidase embedded in nanocarrier PEG shell for enhanced tumor penetration and highly efficient antitumor efficacy. Nano Lett. 2016;16(5):3268-3277.

11. Wang Y, Shim MS, Levinson NS, Sung HW, Xia Y. Stimuli-responsive materials for controlled release of theranostic agents. Adv Funct Mater. 2014;24(27):4206-4220.

12. Luo C, Sun J, Sun B, He Z. Prodrug-based nanoparticulate drug delivery strategies for cancer therapy. Trends Pharmacol Sci. 2014;35(11): 556-566.

13. Yu W, Zhang N, Li C. Saccharide modified pharmaceutical nanocarriers for targeted drug and gene delivery. Curr Pharm Des. 2009;15(32): 3826-3836.
14. Guhagarkar SA, Majee SB, Samad A, Devarajan PV. Evaluation of pullulan-functionalized doxorubicin nanoparticles for asialoglycoprotein receptor-mediated uptake in Hep G2 cell line. Cancer Nanotechnol. 2011;2(1-6):49-55.

15. Xu M, Qian J, Liu X, Liu T, Wang H. Stimuli-responsive PEGylated prodrugs for targeted doxorubicin delivery. Mater Sci Eng C Mater Biol Appl. 2015;50:341-347.

16. Pranatharthiharan S, Patel MD, Malshe VC, et al. Asialoglycoprotein receptor targeted delivery of doxorubicin nanoparticles for hepatocellular carcinoma. Drug Deliv. 2017;24(1):20-29.

17. Medina SH, Tekumalla V, Chevliakov MV, Shewach DS, Ensminger WD, El-Sayed ME. N-acetylgalactosamine-functionalized dendrimers as hepatic cancer cell-targeted carriers. Biomaterials. 2011;32(17): 4118-4129.

18. Kuruvilla SP, Tiruchinapally G, Elazzouny M, Elsayed ME, Elsayed ME. $\mathrm{N}$-acetylgalactosamine-targeted delivery of dendrimer-doxorubicin conjugates influences doxorubicin cytotoxicity and metabolic profile in hepatic cancer cells. Adv Healthc Mater. 2017;6(5):1601046.

19. Chong DQ, Tan IB, Choo SP, Toh HC. The evolving landscape of therapeutic drug development for hepatocellular carcinoma. Contemp Clin Trials. 2013;36(2):605-615.

20. Kalyan A, Nimeiri H, Kulik L. Systemic therapy of hepatocellular carcinoma: current and promising. Clin Liver Dis. 2015;19(2):421-432.

21. Zhu AX. Molecularly targeted therapy for advanced hepatocellular carcinoma in 2012: current status and future perspectives. Semin Oncol. 2012;39(4):493-502.

22. Wilhelm S, Carter C, Lynch M, et al. Discovery and development of sorafenib: a multikinase inhibitor for treating cancer. Nat Rev Drug Discov. 2006;5(10):835-844.

23. Mcdermott U, Sharma SV, Dowell L, et al. Identification of genotypecorrelated sensitivity to selective kinase inhibitors by using high-throughput tumor cell line profiling. Proc Natl Acad Sci U S A. 2007;104(50): 19936-19941.

24. Tai WT, Cheng AL, Shiau CW, et al. Signal transducer and activator of transcription 3 is a major kinase-independent target of sorafenib in hepatocellular carcinoma. J Hepatol. 2011;55(5):1041-1048.

25. Abou-Alfa GK, Johnson P, Knox JJ, et al. Doxorubicin plus sorafenib vs doxorubicin alone in patients with advanced hepatocellular carcinoma: a randomized trial. JAMA. 2010;304(19):2154-2160.

26. Wang C, Su L, Wu C, Wu J, Zhu C, Yuan G. RGD peptide targeted lipid-coated nanoparticles for combinatorial delivery of sorafenib and quercetin against hepatocellular carcinoma. Drug Dev Ind Pharm. 2016;42(12):1938-1944.

27. Kim DH, Kim MD, Choi CW, et al. Antitumor activity of sorafenibincorporated nanoparticles of dextran/poly(dl-lactide-co-glycolide) block copolymer. Nanoscale Res Lett. 2012;7(1):91.

28. Fan X, Zhao X, Qu X, Fang J. pH sensitive polymeric complex of cisplatin with hyaluronic acid exhibits tumor-targeted delivery and improved in vivo antitumor effect. Int J Pharm. 2015;496(2):644-653.

29. Wang H, Sun G, Zhang Z, Ou Y. Transcription activator, hyaluronic acid and tocopheryl succinate multi-functionalized novel lipid carriers encapsulating etoposide for lymphoma therapy. Biomed Pharmacother. 2017;91:241-250.

30. Cui T, Zhang S, Sun H. Co-delivery of doxorubicin and $\mathrm{pH}$-sensitive curcumin prodrug by transferrin-targeted nanoparticles for breast cancer treatment. Oncol Rep. 2017;37(2):1253-1260.

31. Chou TC, Talalay P. Quantitative analysis of dose-effect relationships: the combined effects of multiple drugs or enzyme inhibitors. Adv Enzyme Regul. 1984;22:27-55.

32. Zhang R, Ru Y, Gao Y, Li J, Mao S. Layer-by-layer nanoparticles co-loading gemcitabine and platinum (IV) prodrugs for synergistic combination therapy of lung cancer. Drug Des Devel Ther. 2017;11: 2631-2642.

33. Gao Z, Li Z, Yan J, Wang P, Irinotecan WP. Irinotecan and 5-fluorouracilco-loaded, hyaluronic acid-modified layer-by-layer nanoparticles for targeted gastric carcinoma therapy. Drug Des Devel Ther. 2017;11: 2595-2604. 
34. Zhang P, Li J, Ghazwani M, et al. Effective co-delivery of doxorubicin and dasatinib using a PEG-Fmoc nanocarrier for combination cancer chemotherapy. Biomaterials. 2015;67:104-114.

35. Shao M, Yang W, Han G. Protective effects on myocardial infarction model: delivery of schisandrin B using matrix metalloproteinasesensitive peptide-modified, PEGylated lipid nanoparticles. Int $J$ Nanomedicine. 2017;12:7121-7130.

36. Lee ES, Gao Z, Kim D, Park K, Kwon IC, Bae YH. Super pH-sensitive multifunctional polymeric micelle for tumor $\mathrm{pH}(\mathrm{e})$ specific TAT exposure and multidrug resistance. J Control Release. 2008;129(3): 228-236.

37. Tan S, Wang G, Redox-Responsive WG. Redox-responsive and pHsensitive nanoparticles enhanced stability and anticancer ability of erlotinib to treat lung cancer in vivo. Drug Des Devel Ther. 2017;11 3519-3529.
38. Qu CY, Zhou M, Chen YW, Chen MM, Shen F, Xu LM. Engineering of lipid prodrug-based, hyaluronic acid-decorated nanostructured lipid carriers platform for 5-fluorouracil and cisplatin combination gastric cancer therapy. Int J Nanomedicine. 2015;10:3911-3920.

39. Nazari-Vanani R, Moezi L, Heli H. In vivo evaluation of a self-nanoemulsifying drug delivery system for curcumin. Biomed Pharmacother. 2017;88:715-720.

40. Chou TC, Talalay P. A simple generalized equation for the analysis of multiple inhibitions of Michaelis-Menten kinetic systems. J Biol Chem. 1977;252:6438-6442.

\section{Publish your work in this journal}

Drug Design, Development and Therapy is an international, peerreviewed open-access journal that spans the spectrum of drug design and development through to clinical applications. Clinical outcomes, patient safety, and programs for the development and effective, safe, and sustained use of medicines are the features of the journal, which has also been accepted for indexing on PubMed Central. The manuscript management system is completely online and includes a very quick and fair peer-review system, which is all easy to use. Visit http://www.dovepress.com/testimonials.php to read real quotes from published authors.

Submit your manuscript here: http://www.dovepress.com/drug-design-development-and-therapy-journal 